\title{
The Mechanism of Gas Anesthetics Affecting the Proliferation, Invasion and Metastasis of Osteosarcoma Cells and its Chemotherapy Sensitivity
}

\author{
XIAOYAN ZHANG, P. L. SUN¹ AND Z. H. DONG²*
}

Anesthesiology Department, ' Orthopaedics Department, Affiliated Hospital of Shandong Medical College, Linyi, 276000, ${ }^{2}$ Anesthesiology Department, Shenzhen Samii International Medical Center, Shenzhen, 581000, China

Zhang et al.: Gas Anesthetics Affecting the Osteosarcoma Cells and its Chemotherapy Sensitivity

To explore the mechanism of gas anesthetics affecting osteosarcoma cell proliferation, invasion and metastasis of osteosarcoma cells and its chemotherapy sensitivity. We collected human osteosarcoma specimens from patients undergoing surgery. The expression of epithelial-to-mesenchymal transition process related proteins fibronectin, N-cadherin, E-cadherin and beta-catenin and the expression of apoptosis-related proteins B-cell lymphoma 2, caspase-9 and caspase-3 was analyzed by western blot. The cell proliferation of each group of cells was measured by cell counting kit-8. Transwell test were carried out to detect cell invasion and metastasis. The messenger RNA expression of phosphoinositide 3-kinase/protein kinase $B$ pathway in cells was analyzed by quantitative reverse transcription polymerase chain reaction. The apoptosis was detected by Annexin V-FITC early apoptosis detection kit and cell viability was detected by 3-(4,5-dimethyl-2-thiazolyl)-2,5-diphenyl-2-H-tetrazolium bromide. Sevoflurane decreased the protein expression of $\mathrm{N}$-cadherin and increased the protein expressions of $\mathbf{E}$-cadherin and beta-catenin in a dosedependent manner $(\mathbf{p}<\mathbf{0 . 0 5})$. The low concentration group had decreased cell proliferation at $12 \mathrm{~h}, 24 \mathrm{~h}$, $36 \mathrm{~h}$ and $72 \mathrm{~h}(\mathrm{p}<0.05)$. The cell proliferation of the high concentration group decreased at $24 \mathrm{~h}, 36 \mathrm{~h}$ and $72 \mathrm{~h}(\mathrm{p}<0.05)$. The sevoflurane reduced the number of cell invasion, cell metastasis and decreased the expression of B-cell lymphoma 2 protein in a dose-dependent manner $(p<0.05)$. It increased the protein expressions of caspase-9 and caspase-3, reduced the messenger RNA expressions of phosphoinositide 3-kinase and protein kinase $B$ and increased cell apoptosis and decreased cell viability in a dose-dependent manner $(\mathbf{p}<0.05)$. Gas anesthetic (sevoflurane) inhibited the proliferation, invasion and metastasis of osteosarcoma cells by inactivating the phosphoinositide 3-kinase/protein kinase B pathway and increased the chemotherapy sensitivity of osteosarcoma cells.

Key words: Gas anesthetics, sevoflurane, osteosarcoma, chemotherapy

Osteosarcoma is one of the most common primary bone cancers in children and adolescents. The 5 y survival rate of patients with non-metastatic osteosarcoma has increased to more than $60 \%$ with the improvement of treatment strategies ${ }^{[1]}$. However, due to the invasiveness of osteosarcoma, about half of the patients will have metastasis, which greatly affects their long term survival ${ }^{[2]}$. Surgery is still the first line treatment for patients with solid tumors including osteosarcoma. In recent years, the influence of anesthesia on tumor growth and recurrence after surgery has attracted more and more attention. More and more evidences show that anesthesia may affect the tumor growth and metastasis after surgery by regulating neuroendocrine stress response and immune system of cancer patients $^{[3]}$. Recently, it has been proposed to use volatile anesthetics such as sevoflurane, desflurane and isoflurane to regulate cancer ${ }^{[4]}$. Recent studies have shown that volatile anesthetics, such as sevoflurane and desflurane, have effects on invasion and metastasis of some tumor cells. It is reported that sevoflurane can inhibit the proliferation and metastasis of various cancer cells ${ }^{[5]}$. Studies have proved that sevoflurane has anti-proliferation effect on colon cancer cells ${ }^{[6]}$. Other studies have shown that sevoflurane can inhibit the infiltration of lung cancer cells and prevent the

*Address for correspondence

E-mail: Dongzhaohui0187@126.com 
metastasis of glioma cells ${ }^{[7]}$. In addition, the researchers also proved that sevoflurane and desflurane can damage the release of neutrophil matrix metalloproteinase and reduce the subsequent metastasis of colon cancer cells ${ }^{[8]}$. In addition, the role of anesthetics in regulating the biological process of tumor cells has also been studied. However, the role of sevoflurane in osteosarcoma has not been studied. Therefore, the purpose of this study is to investigate the influence mechanism of gas anesthetics on the proliferation, invasion and metastasis of osteoblast cells and its chemotherapy sensitivity.

\section{MATERIALS AND METHODS}

\section{Clinical organization:}

We collected 30 human osteosarcoma specimens $(2 \times 2 \times 2 \mathrm{~cm}$ in size $)$ from patients undergoing surgery. The experiment was approved by the medical ethics committee of the hospital. Informed consent of patients was obtained before the study.

Isolation and culture of human osteosarcoma cells: Isolation of human osteosarcoma cells by kit was carried out in Iscove modified Dulbecco medium (IMDM, Gibco, Life Technologies, ca, USA) containing 10 $\%(\mathrm{v} / \mathrm{v})$ heat inactivated fetal bovine serum (FBS, Hyclone, UT, USA). $100 \mu \mathrm{g} / \mathrm{ml}$ streptomycin solution (Gibco, Life Technologies, CA, USA) containing 100 $\mathrm{U} / \mathrm{ml}$ benzylpenicillin was used to culture the osteoblast cells in a humid incubator containing $5 \% \mathrm{CO}_{2}$ at $37^{\circ}$. The cells were effectively frozen at different passage times and re-cultured when necessary.

Sevoflurane treatment of cells: Osteosarcoma cells were pretreated with sevoflurane and inoculated into 24-well or 96 -well plates with $3 \times 10^{4}$ cells per well overnight. According to the experimental scheme described earlier, the plate containing the osteosarcoma cells was put into a sealed modular incubator (Thermo Fischer Scientific, Waltham, MA USA) with inlet and outlet connectors. The inlet connector was lined on the anesthesia machine and sevoflurane (Sigma-Aldrich, St. Louis, Missouri, USA) was added into the chamber through a sevoflurane evaporator (Drager Vapor 2000, Luebeck, Germany). In our experiment, cells were exposed to $5 \%$ or $10 \%$ sevoflurane in air $\mathrm{O}_{2}$ mixture. The concentration of sevoflurane in sealed modular incubator was monitored by anesthetic gas monitor (Kobe Riken Company, Japan). Cells in control group were placed in the same chamber with $21 \% \mathrm{O}_{2}$ and 5 $\% \mathrm{CO}_{2}$.

Experimental grouping: According to the experimental requirements, the cultured cells were divided into three groups: control group (cells were cultured in a room containing $21 \% \mathrm{O}_{2}$ and $5 \% \mathrm{CO}_{2}$ ); low concentration group (5\% sevoflurane was used to anesthetize cells during cell culture); high concentration group (10\% sevoflurane was used to anesthetize cells during cell culture).

\section{Experimental methods:}

Detection of cell proliferation by cell counting kit8 (CCK-8): Osteosarcoma cells were inoculated into a 96-well plate $\left(2 \times 10^{5}\right.$ cells/well $)$ and cultured in Roswell Park Memorial Institute Medium (RPMI)1640 medium at $37^{\circ}$ for $0 \mathrm{~h}, 12 \mathrm{~h}, 24 \mathrm{~h}, 36 \mathrm{~h}$ and 72 $\mathrm{h}$ respectively. Cell proliferation was detected by CCK-8 kit according to manufacturer's protocol. The absorbance was measured at a wavelength of $450 \mathrm{~nm}$ using a microplate absorbance reader. The experiment was repeated at least 3 times.

Transwell analysis of invasion and metastasis: Transwell plate (BD Biosciences, Franklin Lakes, NJ, USA) with $8 \mu \mathrm{m}$ hole was used for in vitro invasion assay. At room temperature, the chamber insert was coated with $200 \mathrm{mg} / \mathrm{ml}$ BD Matrigel ${ }^{\mathrm{TM}}$ matrix (BD Biosciences) overnight. Osteosarcoma cells $\left(1 \times 10^{4}\right.$ cells) in RPMI 1640 medium were added to the upper chamber of transwell plate. RPMI 1640 medium containing $20 \%$ FBS was added to the lower chamber as chemical attractant. After incubation for $48 \mathrm{~h}$, the cells were removed from the upper surface with cotton swabs and the infiltrated cells were fixed with methanol and stained with $0.5 \%$ crystal violet at room temperature for $30 \mathrm{~min}$. Images were captured and cells were counted at a magnification of 200 times using an optical microscope (Olympus Corporation, Tokyo, Japan). For metastasis analysis, a $10 \mu 1$ pipette tip was used to scrape the fused monolayer of osteosarcoma cells cultured on a 24-well plate. The wells were washed to remove cell debris and the cell metastasis was allowed for $48 \mathrm{~h}$. Under the light inverted microscope (Olympus; Magnification, x100), representative images were captured. The experiment was repeated at least 3 times.

Western blot method: After the relevant treatment, the total protein in osteosarcoma cells was extracted with Radioimmunoprecipitation assay (RIPA) lysis and extraction buffer (Thermo Fisher Scientific, Waltham, Massachusetts, USA) according to the manufacturer's instructions and protease inhibitor (Roche, Basel, Switzerland) was added. Protein concentration was quantified using $\mathrm{BCA}^{\mathrm{TM}}$ protein detection kit (Thermo Fisher Scientific, Waltham, Massachusetts, USA). 
According to the manufacturer's instructions, the Western blotting system was established by using Bio-Rad Bis-Tris gel system (bio-rad, Shanghai, China). The protein samples were electrophoresed, metastasized to polyvinylidene fluoride (PVDF) membrane (Millipore, MA, USA) and incubated with primary antibody. The primary antibody was prepared in $1 \%$ bovine serum albumin (BSA, Sigma-Aldrich, St. Louis, Missouri, USA) at a dilution of 1:1,000. The primary antibody was incubated with PVDF membrane at $4^{\circ}$ overnight, washed and placed with goat antirabbit (or anti-mouse) Immunoglobulin G H\&L (Horseradish peroxidase (HRP)) secondary antibody in the dark at room temperature for $1 \mathrm{~h}$. After washing, the blots and antibodies carrying PVDF membrane were metastasized to Bio-Rad ChemiDoc ${ }^{\mathrm{TM}}$ XRS system and $200 \mu$ Immobilon Western chemiluminescence HRP substrate was added to cover the membrane surface. Image Lab ${ }^{\mathrm{TM}}$ software (Bio-Rad, Shanghai, China) was used to capture protein signal and band intensity.

Real-time reverse transcription-polymerase chain reaction (RTq-PCR): TRIzol reagent (Invitrogen; Thermo Fisher Scientific, Inc.) was used to extract total RNA from cells. Glyceraldehyde 3-phosphate dehydrogenase (GAPDH) was used as internal control. Complementary DNA (cDNA) was synthesized according to the manufacturer's agreement using PrimeScript $^{\mathrm{TM}}$ RT kit (Takara Bio, Inc., Otsu, Japan). According to the manufacturer's agreement, SYBR Premix Ex Taq ${ }^{\mathrm{TM}}$ (Takara Bio, Inc.) is used for qPCR. The relative expression of each gene was calculated by $2^{-\Delta \Delta \mathrm{Ct}}$ method.

Detection of apoptosis: After transfection, osteoblast osteosarcoma cells in logarithmic growth phase were collected and washed with cold phosphate-buffered saline (PBS) at least 3 times. Annexin V-FITC early apoptosis detection kit (catalog No.6592; Cell Signaling Technology,Inc.) was used for apoptosis analysis. Osteosarcoma cells (1x106) from different groups were resuspended in binding buffer, labeled with $1 \mu$ Annexin V-isothiocyanate (FITC) and $12.5 \mu 1$ propidium iodide (pi) and incubated on ice for $10 \mathrm{~min}$ in darkness. Flow cytometry (FACSCaliburTM; BD Biosciences, Franklin Lakes, NJ, USA) was used for apoptosis analysis. WinMDI software (version 2.5; The Cell Counting Laboratory of Purdue University in West Lafayette, Indiana, USA) was used for data analysis. The experiment was repeated at least 3 times.

Assay of 3-(4,5-dimethyl-2-thiazolyl)-2,5-diphenyl2-H-tetrazolium bromide (MTT): Osteosarcoma cells of all groups were inoculated into $96-$ well plates at a density of $3 \times 10^{4}$ cells $/ \mathrm{ml}$ and cultured at 37 under $5 \% \mathrm{CO}_{2}$ for $48 \mathrm{~h}$. Eight parallel wells were set in each group and $20 \mu \mathrm{l}$ fresh MTT solution (concentration 5 $\mathrm{mg} / \mathrm{ml}$ ) was added to each well. After $4 \mathrm{~h}$ of reaction, $200 \mu \mathrm{l}$ of dimethyl sulfoxide was added to the cells. After full dissolution, the microplate reader was used to detect the optical density (OD) of each group at 490 $\mathrm{nm}$. The experiment was repeated three times and the results were recorded.

Chemical sensitivity test: Cisplatin [cis-diamino (cyclobutane-1,1-dicarboxymethyl) platinum (II)] (DDP) is a recognized cytotoxic agent for solid malignant tumors. $1 \mathrm{mM}$ stock solution (Calbiochem, USA, catalog 232120) was diluted with RPMI medium to achieve $80 \mathrm{mM}$ DDP supplementary medium with clinically relevant concentration. After $24 \mathrm{~h}$, it was restored to standard RPMI medium. Then, MTT assay was used to detect the cell viability of osteoblast osteosarcoma cells. The treated cells were incubated with $20 \mu \mathrm{l}$ MTT solution at $37^{\circ}$ for $4 \mathrm{~h}$. Thereafter, the culture medium was discarded and $150 \mu$ limethyl sulfoxide was added to each well to dissolve the methyl MHz crystal. Cell viability was determined by measuring the absorbance at $570 \mathrm{~nm}$ through a Bio-Tek microplate reader (Bio-Tek, Winooski, USA).

\section{Statistical analysis:}

Data was expressed as mean values (SEM). Tukey postevent multiple comparison test and one-way Analysis of variance (ANOVA) were carried out by using GraphPad Prism (GraphPad Software, La Jolla, CA, USA), except immunocytochemical data, which was subjected to Dunn post-event test by Kruskal-Wallis for nonparametric evaluation. $\mathrm{p}<0.05$ was considered to have statistical significance.

\section{RESULTS AND DISCUSSION}

The expressions of fibronectin, N-cadherin, E-cadherin and $\beta$-catenin in epithelial-to-mesenchymal transition (EMT) process were analyzed by western blot. Compared with control group, the expressions of fibronectin and $\mathrm{N}$-cadherin in low concentration group decreased, while the expressions of E-cadherin and $\beta$-catenin increased $(p<0.05)$. Compared with low concentration group, the protein expressions of fibronectin and $\mathrm{N}$-cadherin in high concentration group decreased, while the protein expressions of E-cadherin and $\beta$-catenin increased $(p<0.05)$, indicating that sevoflurane inhibited epithelial-mesenchymal transition (Table 1). 
www.ijpsonline.com

TABLE 1: WESTERN BLOT ANALYSIS OF EMT-RELATED PROTEINS ( $\overline{\mathrm{x}} \pm \mathbf{s})$

\begin{tabular}{lcccc}
\hline Group & fibronectin & N-cadherin & E-cadherin & B-catenin \\
\hline Control group & $2.62 \pm 0.37$ & $2.45 \pm 0.31$ & $1.14 \pm 0.11$ & $1.37 \pm 0.15$ \\
Low concentration & $1.75 \pm 0.24$ & $1.86 \pm 0.26$ & $1.75 \pm 0.22$ & $2.03 \pm 0.28$ \\
group & $1.03 \pm 0.08$ & $1.14 \pm 0.10$ & $2.38 \pm 0.35$ & $2.64 \pm 0.42$ \\
$\begin{array}{l}\text { High concentration } \\
\text { group }\end{array}$ & 11.026 & 13.695 & 9.525 & 10.036 \\
F value & 0.026 & 0.015 & 0.038 & 0.027 \\
p value & &
\end{tabular}

The cell proliferation of each group at $0 \mathrm{~h}, 12 \mathrm{~h}, 24 \mathrm{~h}, 36$ $\mathrm{h}$ and $72 \mathrm{~h}$ was measured by cell counting kit-8 (CCK8). Compared with control group, low concentration group had the same cell proliferation at $0 \mathrm{~h}$, but the cell proliferation decreased at $12 \mathrm{~h}, 24 \mathrm{~h}, 36 \mathrm{~h}$ and 72 $\mathrm{h}(\mathrm{p}<0.05)$. Compared with low concentration group, high concentration group had the same cell proliferation at $0 \mathrm{~h}$ and $12 \mathrm{~h}$, but the cell proliferation decreased at 24 $\mathrm{h}, 36 \mathrm{~h}$ and $72 \mathrm{~h}(\mathrm{p}<0.05)$, indicating that sevoflurane inhibited cell proliferation (fig. 1, Table 2).

Transwell test was used to detect the invasion and metastasis of cells. Compared with control group, the number of invasion and metastasis in low concentration group decreased $(\mathrm{p}<0.05)$, while that in high concentration group was lower $(\mathrm{p}<0.05)$, indicating that sevoflurane inhibited invasion and metastasis (fig. 2, Table 3).

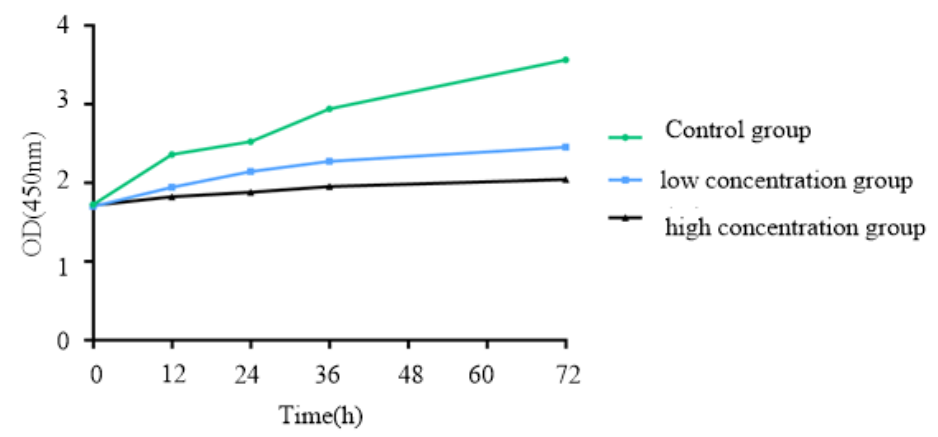

Fig. 1: MTT assay of cell proliferation

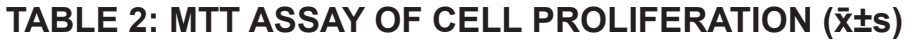

\begin{tabular}{lccccc}
\hline Group & $0 \mathrm{~h}$ & $12 \mathrm{~h}$ & $24 \mathrm{~h}$ & $36 \mathrm{~h}$ & $72 \mathrm{~h}$ \\
\hline Control group & $1.72 \pm 0.13$ & $2.36 \pm 0.26$ & $2.52 \pm 0.28$ & $2.94 \pm 0.31$ & $3.56 \pm 0.40$ \\
$\begin{array}{l}\text { Low concentration } \\
\text { group }\end{array}$ & $1.70 \pm 0.11$ & $1.94 \pm 0.22$ & $2.14 \pm 0.26$ & $2.27 \pm 0.28$ & $2.45 \pm 0.33$ \\
$\begin{array}{l}\text { High concentration } \\
\text { group }\end{array}$ & $1.71 \pm 0.12$ & $1.82 \pm 0.20$ & $1.88 \pm 0.21$ & $1.95 \pm 0.23$ & $2.04 \pm 0.26$ \\
F value & 11.403 & 10.386 & 9.588 & 11.254 & 10.239 \\
p value & 0.504 & 0.037 & 0.024 & 0.017 & 0.004 \\
\hline
\end{tabular}

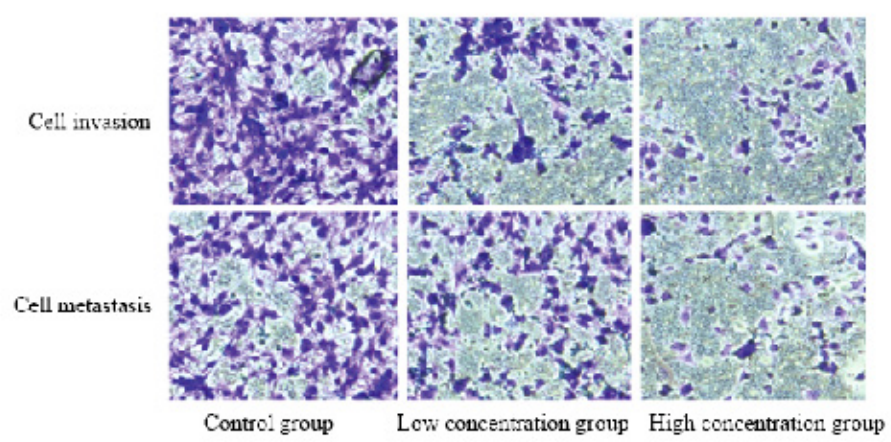

Fig. 2: Assay of cell invasion and metastasis by transwell test 
TABLE 3: ASSAY OF CELL INVASION AND METASTASIS ( $\overline{\mathbf{x}} \pm \mathbf{s})$

\begin{tabular}{lcc}
\hline Group & Cell invasion & Cell metastasis \\
\hline Control group & $317.36 \pm 24.83$ & $278.91 \pm 15.30$ \\
Low concentration group & $262.58 \pm 16.33$ & $184.21 \pm 12.08$ \\
High concentration group & $104.36 \pm 12.58$ & $96.37 \pm 6.15$ \\
F value & 12.392 & 11.467 \\
p value & 0.028 & 0.016 \\
\hline
\end{tabular}

The expressions of apoptosis-related proteins B-cell lymphoma 2 (Bcl-2), caspase-9 and caspase-3 were analyzed by western blot. The expression of Bcl-2 in low concentration group was lower than that in control group, while the expressions of caspase- 9 and caspase- 3 in low concentration group increased $(\mathrm{p}<0.05)$. Compared with low concentration group, the expression of Bcl-2 protein in high concentration group decreased and the expressions of caspase- 9 and caspase- 3 proteins in high concentration group increased $(\mathrm{p}<0.05)$, indicatubg that sevoflurane promoted cell apoptosis (Table 4).

The messenger RNA (mRNA) expression of phosphoinositide 3-kinase (PI3K)/protein kinase B (AKT) pathway in cells was analyzed by Quantitative reverse transcription polymerase chain reaction (RTqPCR). It was found that the mRNA expressions of
PI3K and AKT in low concentration group was lower than those in control group $(p<0.05)$, while those in high concentration group were lower $(\mathrm{p}<0.05)$ (fig. 3 , Table 5).

Cisplatin was used to treat the cells in each group. The apoptosis was detected by Annexin V-FITC early apoptosis detection kit and the cell viability was detected by MTT. Compared with control group, the apoptosis of low concentration group increased, but the cell viability of low concentration group decreased $(p<0.05)$. Compared with low concentration group, apoptosis in high concentration group increased and cell viability in high concentration group decreased $(p<0.05)$, indicating that sevoflurane promoted the chemotherapy sensitivity of cell (Table 6).

TABLE 4: APOPTOSIS PROTEIN EXPRESSION LEVEL ( $\bar{x} \pm \mathbf{s})$

\begin{tabular}{lccc}
\hline Group & Bcl-2 & Caspase-9 & Caspase-3 \\
\hline Control group & $2.05 \pm 0.26$ & $1.04 \pm 0.15$ & $1.25 \pm 0.16$ \\
Low concentration group & $1.44 \pm 0.14$ & $1.74 \pm 0.21$ & $1.94 \pm 0.26$ \\
High concentration group & $0.93 \pm 0.08$ & $2.39 \pm 0.26$ & $2.34 \pm 0.31$ \\
F value & 12.594 & 10.421 & 13.086 \\
p value & 0.027 & 0.016 & 0.033 \\
\hline
\end{tabular}

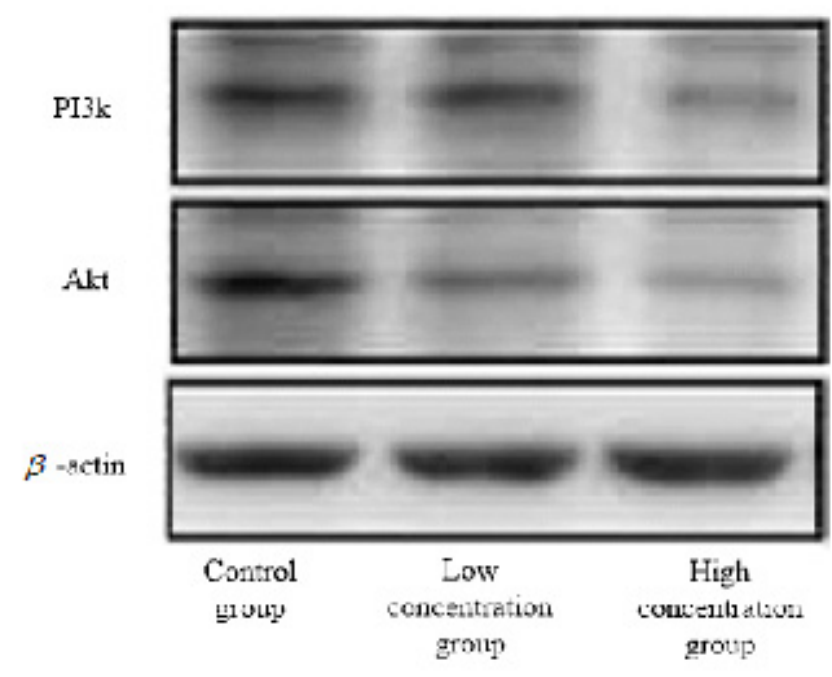

Fig. 3: RT-qPCR analysis 
www.ijpsonline.com

TABLE 5: RT-qPCR ANALYSIS OF EXPRESSION LEVELS OF PI3K/AKT mRNA ( $\bar{x} \pm s)$

\begin{tabular}{lcc}
\hline Group & PI3K & AKT \\
\hline Control group & $2.54 \pm 0.37$ & $2.46 \pm 0.35$ \\
Low concentration group & $1.82 \pm 0.24$ & $1.88 \pm 0.27$ \\
High concentration group & $1.15 \pm 0.13$ & $1.24 \pm 0.15$ \\
F value & 11.382 & 12.085 \\
$\mathrm{p}$ value & 0.025 & 0.017 \\
\hline
\end{tabular}

TABLE 6: ASSAY OF CELL VIABILITY AND APOPTOSIS ( $\bar{x} \pm \mathbf{s})$

\begin{tabular}{lcc}
\hline Group & Cell apoptosis (\%) & Cell viability (\%) \\
\hline Control group & $11.34 \pm 1.53$ & $92.18 \pm 3.46$ \\
Low concentration group & $23.48 \pm 4.35$ & $81.65 \pm 1.33$ \\
High concentration group & $44.16 \pm 1.13$ & $56.87 \pm 1.14$ \\
F value & 12.547 & 13.267 \\
p value & 0.015 & 0.006 \\
\hline
\end{tabular}

Osteosarcoma is a primary bone tumor with high malignant rate, high incidence, high frequency of recurrence and metastasis, especially in children and adolescents. Surgical resection combined with adjuvant therapy is effective for osteosarcoma. With the development of treatment (including chemotherapy and surgery), the survival rate of osteosarcoma has increased to $65-75 \%{ }^{[9]}$. However, due to tumor recurrence and metastasis, the $5 \mathrm{y}$ survival rate of patients is still very low. It is vital important to better understand the pathogenesis of osteosarcoma and determine new treatment strategies and drugs for osteosarcoma. The invasion and metastasis of cancer cells greatly promotes the recurrence of the disease. Recently, anesthetics have been shown to affect the clinical outcome of cancer patients who have undergone surgical resection ${ }^{[10]}$. Sevoflurane is one of the most commonly used volatile anesthetics, which has been proved to inhibit cell proliferation and metastasis in various types of cancers including osteosarcoma ${ }^{[11]}$. In this study, we focused on the anti-proliferation and antiinvasion effects of sevoflurane in osteosarcoma cells and found that sevoflurane played a role in inhibition of osteosarcoma cells.

Sevoflurane has been proved to inhibit the proliferation of cancer cells in various types of cancers, including colorectal cancer, glioma, breast cancer, cervical cancer and osteosarcoma ${ }^{[12]}$. Various surgical operations, such as biopsy and resection are performed for cancer treatment and general anesthesia is usually performed for these procedures. It has been reported that general anesthesia can impair immune response, while surgery can induce cancer cells to spread to surrounding tissues or circulation. These findings indicate that cancer cell infiltration or metastasis is easy to occur during surgery. Studies have shown that surgery may increase the invasion and metastasis potential of cancer cells, thus improving the diffusion ability of cancer cells during perioperative period ${ }^{[13]}$. Therefore, sevoflurane therapy may play an important role in reducing postoperative tumor recurrence. Similarly, it is essential to choose appropriate anesthesia in the process of tumor resection. It is best to choose an anesthetic which can inhibit the proliferation, invasion and metastasis of cancer cells. The exploration of the molecular mechanism related to the effect of anesthesia on proliferation and metastasis of osteosarcoma may pave the way for developing a new method to treat osteosarcoma. Recent studies have shown that anesthetics may affect cancer progression after surgery. In the experiment, to simulate the clinical anesthesia environment, two different concentrations (5\% and $10 \%$ ) of sevoflurane were applied on osteosarcoma cells. We found that sevoflurane inhibited the activity and proliferation of osteoblast cells in a dose-dependent and time-dependent manner.

EMT is an important biological process in tumor metastasis and plays a vital role in invasion and metastasis of tumor cells. This process reduces the expression of cell-cell adhesion molecules (e.g., E-cadherin) and up-regulates the expressions of plastic interstitial proteins (including vimentin, $\mathrm{N}$-cadherin and smooth muscle actin) $)^{[14]}$. EMT process enables tumor cells to acquire higher metastasis characteristics and invasiveness, which is a key step in tumor metastasis process and leads to cancer diffusion and treatment failure. In this study, we found that sevoflurane reduced 
the expressions of $\mathrm{N}$-cadherin and fibronectin in a dosedependent manner and increased the expressions of E-cadherin and $\beta$-catenin. These findings also indicate that sevoflurane can inhibit EMT process in osteoblast cells.

It has been proved that cell invasion and metastasis play an important role in tumor metastasis. Transwell assay was used to study the effect of sevoflurane on invasion and metastasis of osteosarcoma cells. Compared with control group cells, sevoflurane with low concentration of $5 \%$ and high concentration of $10 \%$ inhibited metastasis of osteosarcoma cells. These data indicated that sevoflurane reduced invasion and metastasis of osteoblast osteosarcoma cells. The expressions of apoptosis-related proteins (Bcl-2, caspase-3 and caspase-9) were measured by Western blot. After treated with $5 \%$ and $10 \%$ sevoflurane respectively, the expression of Bcl-2 decreased, while the expressions of caspase-3 and caspase-9 increased in osteoblast cells. These results indicated that sevoflurane promoted apoptosis of osteoblast cells.

PI3K/AKT pathway is one of the most important carcinogenic pathways in human cancer cells. There is evidence that PI3K/AKT signaling pathway is often over-activated in osteosarcoma cells and contributes to tumorigenesis, tumor cell proliferation, invasion, cell cycle progression, apoptosis inhibition, angiogenesis, metabolism and chemical resistance ${ }^{[15]}$. The effects of miR-665 on cognitive dysfunction induced by sevoflurane anesthesia in vivo and in vitro showed that $\mathrm{P} 13 \mathrm{~K} / \mathrm{AKT}$ signaling pathway was inactivated by sevoflurane ${ }^{[16]}$. In our study, sevoflurane inhibited the activation of PI3K/AKT pathway in a dose-dependent manner. This proves that sevoflurane has anti-cancer effect on at least osteosarcoma cells. PI3K/AKT pathway is one of the most important signal transduction pathways in cells related to cancer progression and invasion. Therefore, we studied the effects of sevoflurane with concentrations of $5 \%$ and $10 \%$ on the expressions of PI3K and AKT in osteoblast cells. The results showed that compared with control group, the expressions of PI3K and AKT in osteosarcoma cells decreased after treatment with $5 \%$ or $10 \%$ sevoflurane. These results suggest that the anti-cancer effect of sevoflurane on osteoblast cells may be realized through inhibiting PI3K/AKT pathway.

Cisplatin is a cytotoxic chemotherapeutic commonly used to treat various malignant tumors. These invasion cancers usually develop chemical resistance in clinic, so they were selected for this study. It was found that the chemosensitivity of osteoblast cells increased in a dosedependent manner after being treated with cisplatin.

To sum up, our findings indicate that gas anesthetics can inhibit the proliferation, inactivation and metastasis of osteosarcoma cells by inactivating PI3K/AKT pathway and increase the chemotherapy sensitivity of osteosarcoma cells.

\section{Conflicts of interest:}

The authors declared no conflicts of interest.

\section{REFERENCES}

1. Liu Y, He J, Chen X, Li J, Shen M, Yu W, et al. The proapoptotic effect of formononetin in human osteosarcoma cells: involvement of inactivation of ERK and Akt pathways. Cell Physiol Biochem 2014;34(3):637-45.

2. Wang J, Yang X, Han S, Zhang L. CEP131 knockdown inhibits cell proliferation by inhibiting the ERK and AKT signaling pathways in non-small cell lung cancer. Oncol Lett 2020;19(4):3145-52.

3. Khan I, Kang SC. Apoptotic activity of Lactobacillus plantarum DGK-17-fermented soybean seed extract in human colon cancer cells via ROS-JNK signaling pathway. J Food Sci 2017;82(6):1475-83.

4. Deng N, Li L, Gao J, Zhou J, Wang Y, Wang C, et al. Hsa circ_0009910 promotes carcinogenesis by promoting the expression of miR-449a target IL6R in osteosarcoma. Biochem Biophys Res Commun 2018;495(1):189-96.

5. Yu X, Pang L, Yang T, Liu P. lncRNA LINC01296 regulates the proliferation, metastasis and cell cycle of osteosarcoma through cyclin D1. Oncol Rep 2018;40(5):2507-14.

6. Fei D, Zhang X, Lu Y, Tan L, Xu M, Zhang Y. Long noncoding RNA AFAP1-AS1 promotes osteosarcoma progression by regulating miR-497/IGF1R axis. Am J Transl Res 2020;12(5):2155.

7. Li H, Wang M, Zhou H, Lu S, Zhang B. Long noncoding RNA EBLN3P promotes the progression of liver cancer via alteration of microRNA-144-3p/DOCK4 signal. Cancer Manag Res 2020;12(5):9339.

8. Fei D, Zhang X, Liu J, Tan L, Xing J, Zhao D, et al. Long noncoding RNA FER1L4 suppresses tumorigenesis by regulating the expression of PTEN targeting miR-18a-5p in osteosarcoma. Cell Physiol Biochem 2018;51(3):1364-75.

9. Cai W, Xu Y, Zuo W, Su Z. MicroR-542-3p can mediate ILK and further inhibit cell proliferation, migration and invasion in osteosarcoma cells. Aging (Albany NY) 2019;11(1):18-32.

10. Xie W, Xiao J, Wang T, Zhang D, Li Z. MicroRNA-876-5p inhibits cell proliferation, migration and invasion by targeting c-Met in osteosarcoma. J Cell Mol Med 2019;23(5):3293-301.

11. Jiao J, Wang Y, Sun X, Jiang X. Midazolam induces A549 cell apoptosis in vitro via the miR-520d-5p/STAT3 pathway. Int J Clin Exp Pathol 2018;11(3):1365-73.

12. Liu Y, Zhang J, Xing C, Wei S, Guo N, Wang Y. miR486 inhibited osteosarcoma cells invasion and epithelialmesenchymal transition by targeting PIM1. Cancer Biomark 2018;23(2):269-77.

13. Cai W, Xu Y, Yin J, Zuo W, Su Z. miR-552-5p facilitates osteosarcoma cell proliferation and metastasis by targeting WIF1. Exp Ther Med 2019;17(5):3781-8. 
14. Cai W, Xu Y, Yin J, Zuo W, Su Z. miR-590-5p suppresses osteosarcoma cell proliferation and invasion via targeting KLF5. Mol Med Rep 2018;18(2):2328-34.

15. Zang J, Zuo D, Shogren KL, Gustafson CT, Zhou Z, Thompson MA, et al. STIM1 expression is associated with osteosarcoma cell survival. Chin J Cancer Res 2019;31(1):203-11.

16. Wei G, Zhang T, Li Z, Yu N, Xue X, Zhou D, et al. USF1mediated upregulation of IncRNA GAS6-AS2 facilitates osteosarcoma progression through miR-934/BCAT1 axis. Aging (Albany NY) 2020;12(7):6172-490.
This is an open access article distributed under the terms of the Creative Commons Attribution-NonCommercial-ShareAlike 3.0 License, which allows others to remix, tweak, and build upon the work non-commercially, as long as the author is credited and the new creations are licensed under the identical terms

\begin{tabular}{l} 
This article was originally published in a special issue, \\
"Therapeutic Perspectives in Biomedical Research and Pharma- \\
ceutical Sciences and their Nursing Methods" \\
Indian J Pharm Sci 2021:83(4)Spl issue "190-197" \\
\hline
\end{tabular}

